\title{
Giovanni VerGA
}

La vida en el campo. Trad. in castigliano di Hugo Bachelli

Cáceres: Periférica, 2008, 160 p.

Da tempo si sentiva il bisogno di una traduzione in ambito ispanico di Vita dei campi dopo le lontane edizioni di Cipriano Rivas Cherif, Ruíz Sommers, José Valbuena.

A questo non facile compito si è sottoposto, recentemente, Hugo Bachelli.

Il suo volumetto, pubblicato con il patrocinio della «Consejería de Cultura y Turismo de la Junta de Extremadura» contiene la traduzione delle otto novelle che formano Vita dei campi e che furono pubblicate dal Verga su varie riviste (Fanfulla della domenica, Il Fanfulla, Rivista minima, La fronda), tra l'agosto 1878 e il luglio 1880: Fantasticheria, Jeli il pastore, Rosso Malpelo, Cavalleria rusticana, La Lupa, Lamante di Gramigna, Guerra di Santi, Pentolaccia.

Il Bachelli, nell'intento di far traduzione castigliana accessibile a tutti, anche ai non letterati, come ci dice nella «nota finale del traduttore», ha ritenuto di abolire molte parti nelle varie novelle e di staccarsi il più possibile dall'originale verghiano. Sempre nella nota alla fine del librino, scrive di aver consultato alcune fra le edizioni più conosciute (Mondadori, Salerno, Rizzoli, Feltrinelli) anche se ammette di aver attinto a "tutte le versioni apparse, durante quasi un secolo, su varie riviste, giornali e libri». Ci sembra, comunque, strano che tali pubblicazioni manchino di parti così sostanziali rispetto alle edizioni più conosciute e studiate dalla critica verghiana.

$\mathrm{Ne}$ esce una traduzione che non ha il pregio di un'aderenza assoluta e agile al testo e neanche quello di conservare l'andamento e il tono dell'originale italiano. Difetta di quel sintetico dinamismo verbale che è la prerogativa del Verga in quanto il Bachelli non riesce a trasferire al castigliano la sintassi breve e concisa dell'autore né lo stile teso e incisivo capace a cogliere la problematica sociale dell'ambiente contadino e cittadino, così come il tragico baratro della condizione umana. Inoltre la traduzione presenta imprecisioni e sviste.

Si confrontino questi passi delle novelle Pentolaccia, La Lupa, Cavalleria rusticana, Guerra di Santi. con la versione castigliana [cito sempre dall'edizione A. Mondadori, 1990, p.226]:

«Per disgrazia Venera non se ne accorse, perché in quel momento era andata in cucina a mettere una bracciata di legna sotto la caldaia che bolliva. Appena don Liborio mise piede nella stanza, suo compare levò la stanga, e gli lasciò cadere fra capo e collo tal colpo, che l'ammazzò come un bue, senza bisogno di medico, né di speziale».

«Venera, que había ido a la cocina para echar otra brazada de leña a la lumbre no se dio cuenta de nada. No vio a su marido escondido tras la puerta, no vio cómo don Liborio entraba en la casa, no vio cómo el palo le abría la cabeza como si fuera la de un buey, de tal manera que no fueron necessarios ni médico ni cura».

«Nei campi immensi, dove scoppiettava soltanto il volo dei grilli, quando il sole batteva a piombo, la Lupa, affastellava manipoli su manipoli, e covoni su covoni, senza stancarsi mai, senza rizzarsi un momento sulla vita, senza accostare le labbra al fiasco, pur di stare sempre alle calcagna di Nanni, che mieteva e mieteva, e le domandava di quando in quando: "Che volete, gnà Pina?"”. [p.198]

En los campos inmensos, donde sólo se oía el canto de los grillos, cuando caía el sol a plomo, la loba agavilla- 
ba manojo, y haz tra haz, sin cansarse jamás, sin alzar un momento el cuerpo, sin acercar los labios a la botella, con tal de estar siempre cerca de Nanni, que segaba y segaba, y le preguntaba de vez en cuando:

- ¿Qué quiere, señora Pina?

Ella se ne andava infatti, la Lupa, riannodando le trecce superbe, guardando fisso dinanzi ai suoi passi nelle stoppie calde, cogli occhi neri come il carbone. [p. 199]

La Loba se hizo otra vez aquellas magníficas trenzas y se fue, contando cada paso, por los calientes rastrojos.

«La gnà Lola si maritò col carrettiere; e la domenica si metteva sul ballatoio, colle mani sul ventre per far vedere tutti i grossi anelli d'oro che le aveva regalati suo marito».

[p. 191]

«Lola se casó con el carretero. Los domingos se ponía en el atrio de la iglesía con los brazos en jarras para enseñar todos los añillos de oro que le había regalado su marido».

"Compare Alfio era di quei carrettieri che portano il berretto sull'orecchio, e a sentir parlare in tal modo di sua moglie cambiò di colore come se l'avessero accoltellato. - Santo diavolone! esclamò, se non avete visto bene, non vi lascierò gli occhi per piangere! a voi e a tutto il vostro parentado!» [p.194]

"Alfio era uno de esos carreteros que calan su boina hasta las orejas, con la mirada, come allí dicen, «sin fondos», y al oír hablar de su mujer de aquel modo cambió de color como si le hubiesen dado una puñalada. Trató de dominarse, pero no pudo.

— ¡Maldita sea! —exclamó-. Eso sí, como te hayas equivocado, no os dejo ojos para llorar ni a ti ni a tu familia».
"-Vogliamo fare una gran festa per ringraziare San Pasquale di averci salvato dal colera. D'ora innanzi non ci saranno più arruffapopoli, né oppositori, ora che è morto quel vice-pretore che ha lasciato la lite nel testamento.

- Sì, la festa per quelli che sono morti! —-sogghignò Nino

- E tu che sei vivo per San Rocco forse?

—La volete finire, saltò su Saridda, che poi ci vorrà un altro colera per far la pace!» [p. 220]

"-Tenemos que hacer una fiesta, para agradecer a san Pasquale que nos haya salvado del cólera.

- Sí, haremos la fiesta por todos los que han muerto - sugirió Nino. - ¿Es que tú estás vivo gracia a tu San Rocco? - dijo Turi.

— ¡Se acabó! — soltó Saridda—. A ver si va a ser necesario otro cólera para hacer las paces».

Il testo del Bachelli poi, non presentando alcuna nota e commento, presuppone-palese contrasto- nel lettore comune una conoscenza approfondita della storia italiana oltre che del Verga e della Sicilia. Infatti a chi non soddisfi queste condizioni la traduzione potrà sembrare oscura e incompleta. Occorreva fornire al lettore straniero un esauriente apparato di note che corredassero il testo delle necessarie spiegazioni storico-linguistiche, metessero in luce i passaggi cruciali dell'operazione narrativa e suggerissero le interpretazioni dei significati fondamentali (frasi idiomatiche, proverbi ecc.). Tutto questo avrebbe permesso di capire meglio i riferimenti o le allusioni che i personaggi affidano alle loro parole.

Andrebbero presi in considerazione gli elementi etnico-geografici presenti nell'humus storico degli autori siciliani e la diversa indole mentale. Scientifico-speculativa in quelli della Sicilia occidentale (Trapani, Palermo) in omaggio alla tra- 
dizione degli arabi, completamente letteraria negli scrittori della Sicilia orientale (Messina, Catania, Siracusa, Agrigento) dove si direbbe aleggi ancora uno spirito della Grecia antica. D'altra parte è impossibile comprendere ed apprezzare l'opera di Verga senza possedere una particolare attitudine a cogliere e percepire le differenze che si possono riscontrare nel dialetto siciliano e che derivano quasi esclusivamente dalla maggiore o minore presenza di relitti arabi e greci. Il lessico latino presenta in tutta l'isola tale uniformità quale di rado è dato di constatare nel resto d'Italia. In Sicilia non si è sempre parlato e non si parla tuttavia, unicamente il siciliano.

Già nell'antichità greco-romana i siciliani parlavano correttamente tre lingue: il greco, il latino e il punico e fino all'età di Augusto le monete siciliane avevano iscrizioni in greco. Sotto i romani e gli svevi l'isola divenne altresì paese di colonizzazione: si giustificano così le isole linguistiche, come Piazza Armerina, Nicosia, che conservano il loro dialetto gallo-italico o quelle che conservano forti tracce di linguaggio settentrionale come Randazzo e Bronte, dovuto, nell'undicesimotredicesimo secolo, alle notevoli immigrazioni dall'Italia settentrionale di soldati e coloni che desideravano abbandonare le terre del Nord colpite dalle lotte comunali.

Le stratificazioni linguistiche più notevoli nel dialetto siciliano sono evidenti anche ai giorni nostri e possono suddividersi in cinque stratificazioni fondamentali: la greco-classica, la greco-bizantina, l'araba, la franco-latina del periodo normanno e la catalano castigliana del periodo aragonese spagnolo; e in talune stratificazioni minori, come l'anglosassone, fino ad arrivare agli americanismi importati in Sicilia nel 1943-45 dalle truppe di occupazione.

Vorremmo anche attirare l'attenzione sull'influsso greco-classico ancora evidente nell'uso che i siciliani fanno del passato remoto invece del passato prossimo, per indicare un fatto recentemente accaduto. Sono poi vocaboli di derivazione greco-classica cannata (anfora), naca (culla), mentre l' influenza greco-bizantina è soprattutto notevole nei toponimi, come nel caso di Adrano diventato Adernò.L' influsso arabo è chiarissimo in un numero notevole di toponimi, come: giarra (giara) da "giarrah, sciarra (rissa) da «sciarrah" e tanti altri. Numerosi sono gli influssi castigliano-catalani del periodo aragonese e spagnolo. L'influsso castigliano da capiri per esservi contenuto, starvi, truppicari per inciampare, scupetta per fucile, ecc. Per influsso catalano si ha il siciliano, attrassari per attardarsi, abbuccari per versare, e molti altri se ne potrebbero elencare.

$\mathrm{Ci}$ sia consentito fare ora qualche personale considerazione. Che il Verga non sia facile da capire e tradurre è universalmente risaputo e ben lo sperimentò lo svizzero Edouard Rod, traduttore di molte opere di Verga al francese che dovette far tesoro delle numerose lettere che gli scrisse l'autore per spiegargli,via via, quelle parti in cui il carattere e l'indole degli innesti fraseologici siciliani mal trovavano una adeguata traduzione. [Cfr. Giovanni Verga, Lettere al suo traduttore, a cura di Fredi Chiappelli, Firenze, Le Monnier, 1954]

Soffermiamoci, per esempio, sull'uso del «voi» in Verga. I sistemi pronominali dell'italiano e dello spagnolo non sono identici, specialmente per quel che riguarda i pronomi allocutori di cortesia. Nelle novelle appare come pronome allocutivo la forma voi con chiara funzione polivalente.Una è la forma al singolare che sta a metà tra lo spagnolo «tú» e «Usted» e viene usata per rivolgersi a persone con le quali non si ha abastanza confidenza per dar loro del tu, persone spesso di livello più elevato ma anche verso persone $\mathrm{di}$ categoria inferiore, subalterni, con lo scopo di stabilire una distanza. La forma Lei, come ben indica il Migliorini nella 
sua Storia della lingua italiana [Firenze, Sansoni, 1983] non riusci ad imporsi nell'ambito del linguaggio popolare e, specialmente, nel sud d'Italia, si continuò ad usare il voi come forma pronominale in singolare. Il voi può inoltre sostituire il pronome di terza persona plurale Loro, spesso considerato troppo formale, né va dimenticato che voi traduce anche il vosotros spagnolo.

La pluralità delle funzioni della forma «voi» non può, pertanto, essere ridotta in castigliano a una forma unica per esprimere i differenti registri che intercorrono tra i personaggi delle varie novelle. Il traduttore deve necessariamente giostrarsi tra le relazioni personali e sociali che intercorrono tra i personaggi, senza dimenticare di considerare le differenze sociali ed economiche che li legano.

Quanto poi alla non traduzione, da parte del Bachelli, del siciliano "gnà», aferesi del "dueña» spagnolo che dà "gnà» per le donne di bassa condizione e "donna» per le donne alle quali si dà del "vossia» e del "voscienza», intenderemmo più che adeguato l'equivalente castigliano señá, giustificando questa interpreta- zione con l'uso che ne fece in Galizia regione molto simile, per povertà ed emigrazione, alla Sicilia - lo scrittore Alfonso Rodríguez Castelao quasi coetaneo del Verga. Nel suo libro Cousas usa spesso «siña» come ipocorístico per señora. Cito a memoria dal racconto Todos cantos sabian algo da hestoria da vila:

... Nos días mainos a siña Sinforosa rezaba polos afogados. O barco ía andando cara á banda de alá e a mandadeira ía rezando sempre.

Si può concludere affermando che il traduttore non ha saputo riscrivere gli elementi dialettali presenti nelle novelle e in generale nella lingua del Verga ricca di varianti lessicali, allotropi e allomorfi né sfruttare la maggiore ricchezza dei registri informali e gergali del castigliano rispetto all'italiano.

Uno scrittore come Verga dovrebbe incutere rispetto e obbligare i traduttori a un previo studio, accurato e filologico, delle sue opere.

Santa Ferretti

\section{Giani STUPARICH}

La isla

Traducción de J. Á. González Sainz

Presentación de Elvio Guagnini. Posfacio de Claudio Magris

Barcelona: Editorial Minúscula, 2008, 119 p.

Un'ottima scelta, come confermano i riconoscimenti della critica e del pubblico, quella di pubblicare questo piccolo gioiello della letteratura triestina e di uno scrittore, qui, pressoché sconosciuto. Giani Stuparich fa parte, insieme ad Italo Svevo, Scipio Slataper e Umberto Saba, tutti accomunati, come le sorelle Malfenti de La coscienza di Zeno, dalla stessa consonante iniziale, di una pattuglia di autori triestini che hanno dato lustro alla prima metà del Novecento italiano. In una post- fazione all'edizione spagnola de L'isola, Claudio Magris, altro grande triestino, inquadra i quattro scrittori, in due opposti schieramenti, quelli appartenenti ad una "triestinidad negra», pervasa da un senso di morte e di pessimismo, che accomuna Slataper e che "serpentea a lo largo de la enfermedad sveviana o de los exorcismos del último Saba». E l'altro in cui si trova Stuparich che «representa el reverso de ese lado oscuro", ovvero una sorta di «triestinidad blanca, o sea, sana, posi- 